\title{
Identifying the most suitable endogenous control for determining gene expression in hearts from organ donors
} Silvia Pérez*1, Luis J Royo², Aurora Astudillo ${ }^{3}$, Dolores Escudero ${ }^{1,4}$, Francisco Álvarez ${ }^{5}$, Aida Rodríguez ${ }^{2}$, Enrique Gómez ${ }^{2}$ and Jesús Otero ${ }^{1}$

\begin{abstract}
Address: ${ }^{1}$ Unidad de Coordinación de Trasplantes y Terapia Celular, Hospital Universitario Central de Asturias, C/Celestino Villamil s/n, 33006 Oviedo, Spain, 22́rea de Genética y Reproducción Animal, SERIDA-Somió, C/Camino de los Claveles 604, 33203 Gijón, Spain, ${ }^{3}$ Servicio de Anatomía Patológica, Hospital Universitario Central de Asturias, Oviedo, Spain, ${ }^{4}$ Servicio de Medicina Intensiva, Hospital Universitario Central de Asturias, Oviedo, Spain and ${ }^{5}$ Servicio de Bioquímica Clínica, Hospital Universitario Central de Asturias, Oviedo, Spain
\end{abstract}

Email: Silvia Pérez* - silvia.perez@sespa.princast.es; Luis J Royo - ljroyo@serida.org; Aurora Astudillo - astudillo@hca.es; Dolores Escudero - lolaescudero@telefonica.net; Francisco Álvarez - falvarez@arrakis.es; Aida Rodríguez - airodriguez@serida.org; Enrique Gómez - egomez@serida.org; Jesús Otero - jesus.otero@sespa.princast.es

* Corresponding author

Published: 20 December 2007

BMC Molecular Biology 2007, 8: I 14 doi:I0.1 I86/I47I-2199-8-II4
Received: 8 May 2007

Accepted: 20 December 2007

This article is available from: http://www.biomedcentral.com/I47/-2/99/8/II4

(c) 2007 Pérez et al; licensee BioMed Central Ltd.

This is an Open Access article distributed under the terms of the Creative Commons Attribution License (http://creativecommons.org/licenses/by/2.0), which permits unrestricted use, distribution, and reproduction in any medium, provided the original work is properly cited.

\begin{abstract}
Background: Quantitative real-time reverse transcription PCR (qRT-PCR) is a useful tool for assessing gene expression in different tissues, but the choice of adequate controls is critical to normalise the results, thereby avoiding differences and maximizing sensitivity and accuracy. So far, many genes have been used as a single reference gene, without having previously verified their value as controls. This practice can lead to incorrect conclusions and recent evidence indicates a need to use the geometric mean of data from several control genes. Here, we identified an appropriate set of genes to be used as an endogenous reference for quantifying gene expression in human heart tissue.

Results: Our findings indicate that out of ten commonly used reference genes (GADPH, PPIA, ACTB, YWHAZ, RRNI8S, B2M, UBC, TBP, RPLP and HPRT), PPIA, RPLP and GADPH show the most stable gene transcription levels in left ventricle specimens obtained from organ donors, as assessed using geNorm and Normfinder software. The expression of TBP was found to be highly regulated.

Conclusion: We propose the use of PPIA, RPLP and GADPH as reference genes for the accurate normalisation of qRT-PCR performed on heart tissue. TBP should not be used as a control in this type of tissue.
\end{abstract}

\section{Background}

Gene expression analysis is a useful technique for determining and comparing gene expression levels in healthy and diseased tissues. One of the most commonly used tools in the area of gene expression quantification is quantitative real-time reverse transcription PCR (qRT-PCR). When small amounts of nucleic acids are available, qRT-
PCR is especially suitable and provides simultaneous measurement of gene expression in many different samples. If we compare this technique with others such as in situ hybridisation, qRT-PCR offers several advantages: it is not time-consuming, only a small amount of tissue is required, it can be used in high throughput systems and no post-reaction manipulation is needed. However, the 
use of qRT-PCR requires compensation for differences between samples, arising from the varying quality and quantity of the starting material, especially when starting with solid tissue, due to the method of RNA extraction and cDNA synthesis [1]. Normalisation should include endogenous control genes (reference genes), and some of the most frequently used reference genes are housekeeping genes. The ideal endogenous control should be expressed at a constant level in the different tissues of an organism at all stages of development and should be unaffected by experimental treatments. It should also be constitutively expressed in the same tissue under different circumstances. There is, however, no universal control gene that is expressed at a constant level under all conditions and in all tissues. Hence, experimental treatments [2] and hormonal stimulation [3], as well as the different methods used to process tissue specimens [4] can induce changes in the expression of typical housekeeping genes.

Consequently, the success of the technique used depends on the adequate choice of the appropriate reference genes. Despite many qRT-PCR studies having reported the use of a single endogenous control [5], a normalisation strategy based on a single housekeeping gene can lead to erroneous results [6,7]. Vandesompele et al. [6] propose the use of a panel of putative reference genes on a representative number of samples, to identify the most stable of these and then establish the optimal number of genes required for the reliable normalisation of RT-PCR data. In the present study, we tested ten commonly used reference genes (GADPH, PPIA, ACTB, YWHAZ, RRN18S, B2M, $U B C, T B P, R P L P$ and HPRT) of different functional classes (significantly reducing the chance that the genes will be co-regulated) in heart tissue obtained from organ donors. Using geNorm software [6], we were able to assess gene expression stability under our experimental conditions and determine how many reference genes were needed for accurate normalisation. Then, by comparing these results with those generated by a similar programme, Normfinder $[8,9]$, we identified a set of reference genes that offers reliable results for qRT-PCR data normalisation for use in gene expression studies involving heart tissue from brain-dead multiorgan donors.

\section{Results}

Thirty five samples of left ventricular tissue were obtained from 35 multiorgan donors. RNA was successfully isolated and cDNA synthesised from all these specimens. All the samples analysed showed a single $\beta$-actin band in the $2 \%$ agarose gel stained with ethidium bromide at the expected size (data not shown), confirming the total absence of residual DNA.

In each sample, qRT-PCR using Sybr Green was performed for ten frequently-used reference genes (GADPH, PPIA,
ACTB, YWHAZ, RRN18S, B2M, UBC, TBP, RPLP and $H P R T)$. The accuracy of the qRT-PCR was assessed by melt curve analysis and gel electrophoresis. Gene-specific amplification was verified, by both a single peak in the melt curve and a single band in the agarose gel, for the 10 genes analysed in the 35 cDNA samples. Correlation coefficients $\left(\mathrm{R}^{2}\right)$ ranged from 0.995 to 0.999 and PCR efficiencies from $89.7 \%$ to $104 \%$. Using the Proc VARCOMP in the SAS/STAT ${ }^{\mathrm{TM}}$ software [10], the reproducibility of the assay was assessed using as control material samples obtained by pooling the whole set of samples analysed in the present study. The high average correlation coefficient observed of 0.998 indicated good intra-sample reproducibility. Observed $\mathrm{Ct}$ values for each gene were similar across different samples indicating low variability $(21.66$ \pm 1.39 for GADPH, $24.69 \pm 1.22$ for PPIA, $22.32 \pm 2.03$ for ACTB, $26.39 \pm 1.36$ for YWHAZ, $11.71 \pm 1.10$ for RRN18S, $23.87 \pm 1.37$ for $\mathrm{B} 2 \mathrm{M}, 22.71 \pm 1.88$ for $\mathrm{UBC}, 30.98 \pm 3.45$ for TBP, $22.27 \pm 1.42$ for RPLP and $28.08 \pm 0.94$ for HPRT) (Figure 1).

The GeNorm software also provided a rank order of the ten candidate reference genes according to their $\mathrm{M}$ values (Figure 2). PPIA, RPLP and GADPH were the three most stable genes. $M$ values increased moderately for all genes, while TBP abruptly attained the highest $M$ value. The pairwise variation $(\mathrm{V})$ upon normalisation with the two most stable genes and introduction of the third one was 0.236. This value decreased gradually until the addition of the fifth gene, when the trend became more or less stable but increased considerably upon the incorporation of the tenth gene (Figure 3).

Table 1 provides the results of the Normfinder analysis performed on our data. The reference genes tested were ordered according to their stability values. Thus, the most stable single gene was GADPH with a stability value of 0.345 and the least stable was TBP with a value of 2.287 .

\section{Discussion}

Human heart tissue specimens are very difficult to acquire. However, we managed to obtain 35 samples from the left ventricles of 35 organ donors of different age, weights and sex, whose hearts could not be used for transplant purposes for various reasons (see Methods). Thus, we can assume that the number and type of samples used was sufficiently high and diverse to ensure their representativity and randomness. Reports of the use of qRT-PCR in human heart tissue are scarce because of the technical difficulties involved in obtaining such samples. However, given that qRT-PCR is especially suitable for determining gene expression in small pieces of tissue, we tried to establish guidelines for accurate data normalisation intended for human heart studies based on qRT-PCR. 


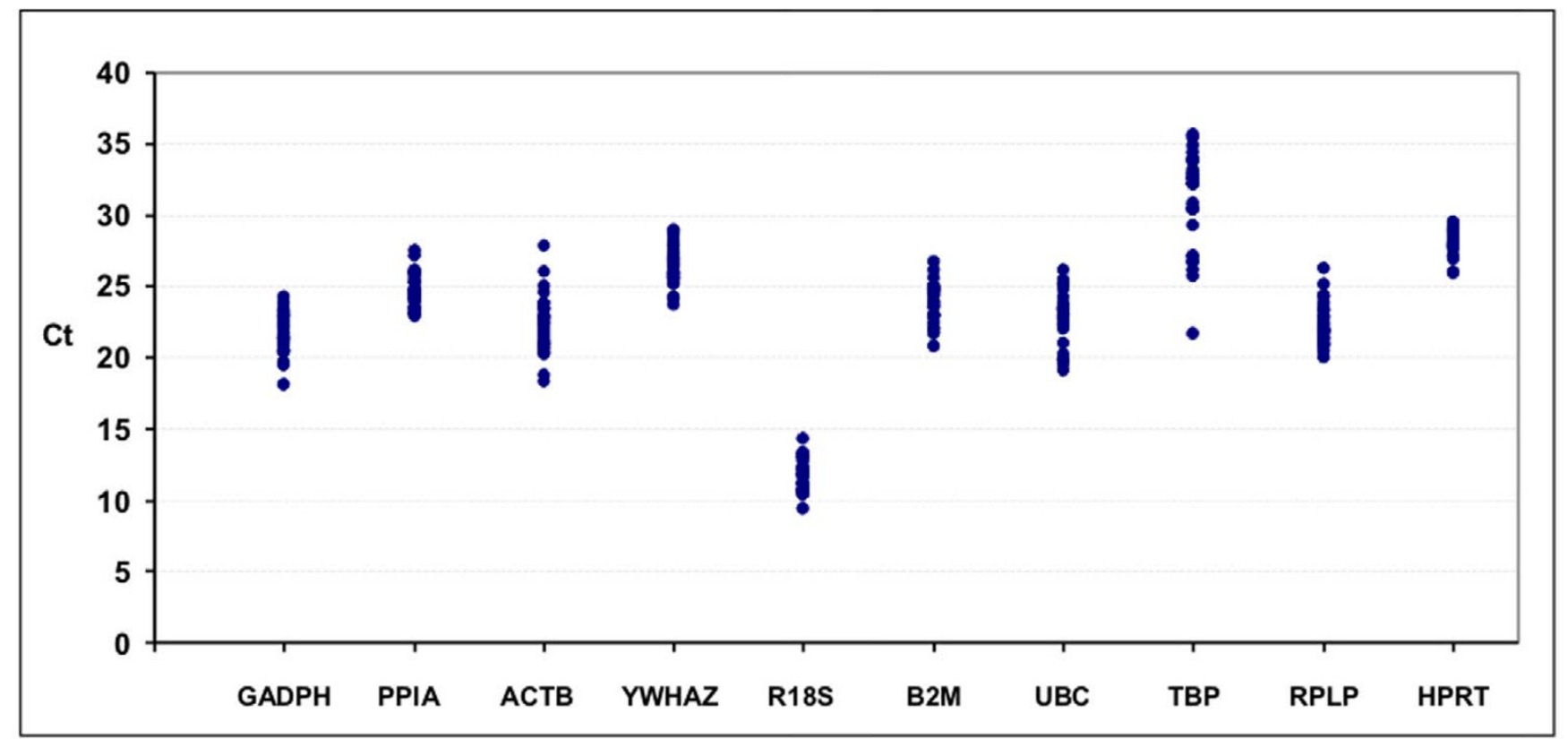

Figure I

Expression levels of candidate housekeeping genes. Scatter plots showing the expression levels of the different reference genes in the tested heart samples $(n=35)$. Values are given as cycle threshold numbers $(C t$ values).

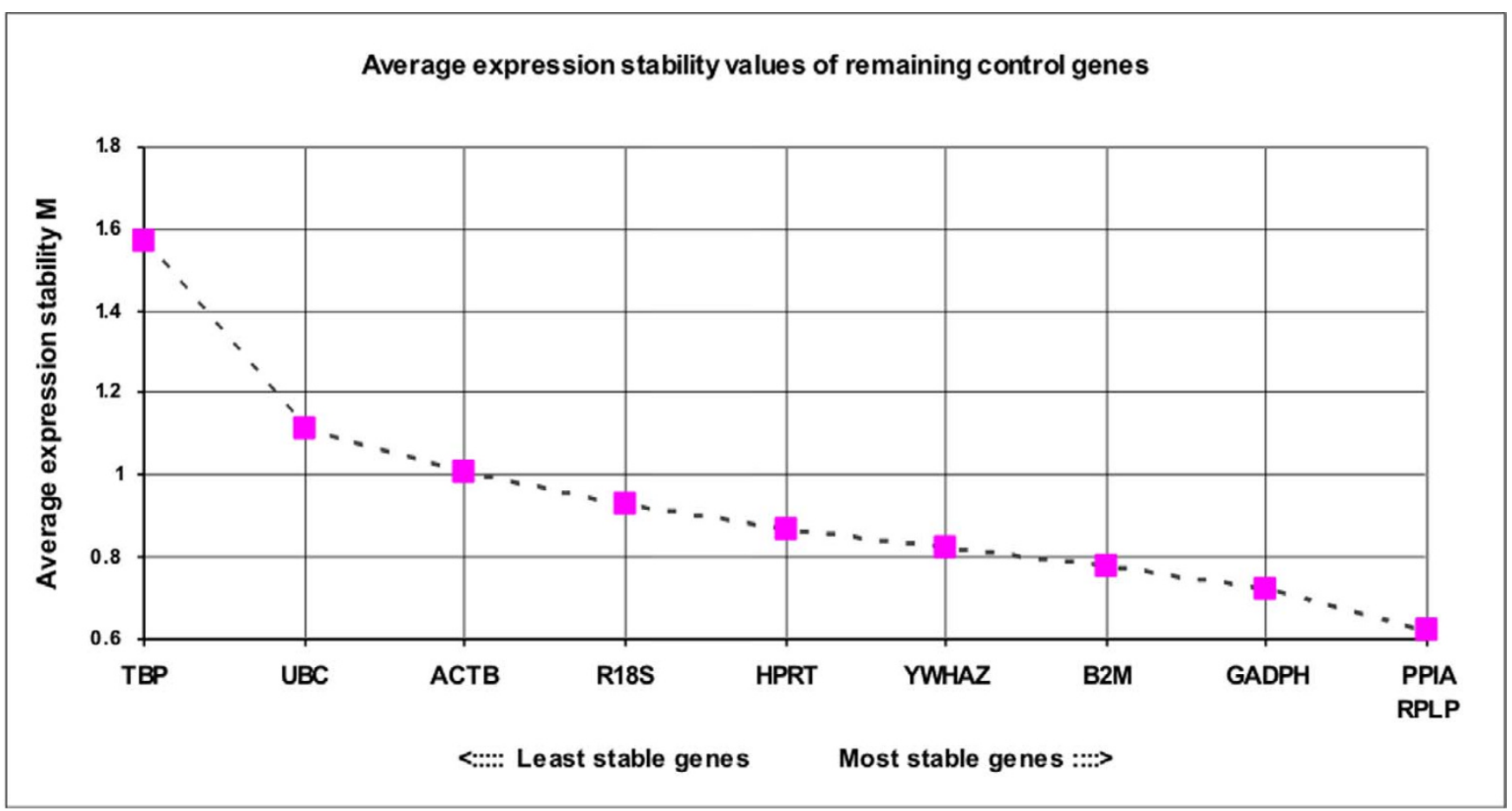

Figure 2

Average expression stability values $(M)$ of the candidate reference genes. Average expression stability measure ( $M$ ) of control genes during stepwise exclusion of least stable reference genes. $M$ is represented from the least stable (left) to the most stable (right), analysed by the geNorm programme. 


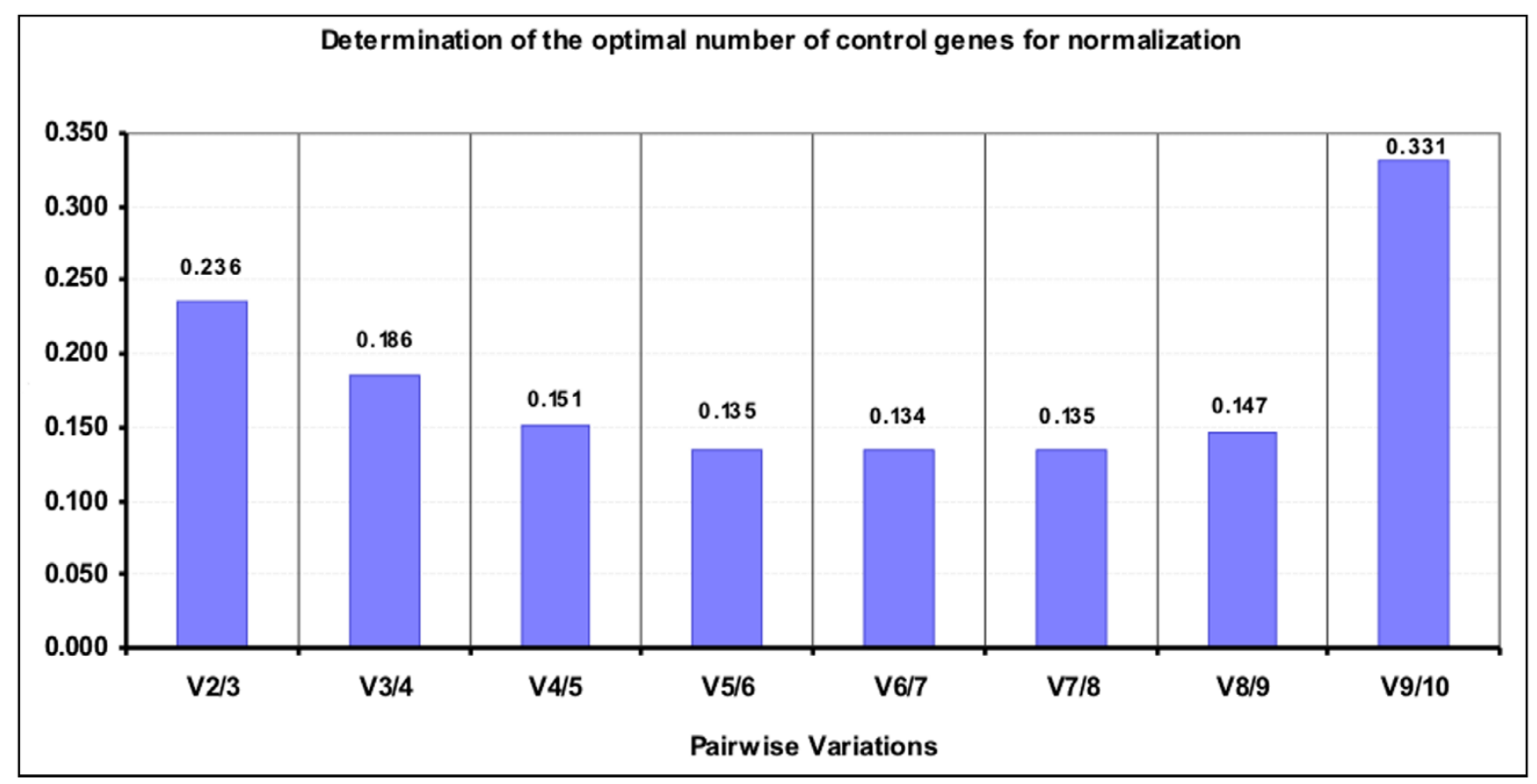

Figure 3

Pairwise variation analysis between normalisation factors to determine the optimal number of control genes for normalisation. Optimal number of control genes for normalisation calculated on the basis of pairwise variation ( $V$ ) analysis. The highest $\mathrm{V} 9 / \mathrm{I} 0$ value is due to the inclusion of a relative unstable gene and is in accordance with the average expression stability (M).

Our technical procedure proved to be a valid method of quantifying gene expression, rendering high correlation coefficients $\left(\mathrm{R}^{2}\right)$ and robust PCR efficiencies.

Through geNorm analysis, PPIA, RPLP and GADPH appeared as the most stable genes and TBP the least stable (Figure 2). It has been reported that in over $90 \%$ of cases, gene expression data are normalised using GADPH, ACTB, $18 S$ rRNA or $28 S$ rRNA as single control genes [5]. However, several studies have shown that these reference genes

Table I: Tested reference genes for normalisation of qRT-PCR listed according to their expression stability calculated by the Normfinder software.

\begin{tabular}{ccc}
\hline Raking Order & Gene Name & Stability Value \\
\hline I & GADPH & 0.345 \\
2 & PPIA & 0.405 \\
3 & RPLP & 0.426 \\
4 & B2M & 0.446 \\
5 & YWHAZ & 0.498 \\
6 & HPRT & 0.588 \\
7 & RRNI8S & 0.593 \\
8 & ACTB & 0.737 \\
9 & UBC & 0.831 \\
10 & TBP & 2.287 \\
\hline
\end{tabular}

undergo variation according to the experimental conditions, treatment and cell cycle stage $[11,12]$.

In human adipocytes and preadipocytes, $A C T B$ and $18 \mathrm{~S}$ $r R N A$ gene expression levels change under hormonal stimulation [3]. Moreover, it has been reported that ACTB is unsuitable as a control for gene expression analysis in interstitial cells derived from sheep heart valves [13]. The $18 S$ rRNA gene has been considered an ideal internal control in qRT-PCR analysis. However, ribosomal RNA accounts for up to $80-90 \%$ of total cellular RNA, and several studies have shown that rRNA varies less under conditions that affect the expression of mRNAs [14] but that possible imbalances in rRNA and mRNA fractions between different samples makes genes encoding ribosomal RNAs unsuitable as references [6]. As far as we are aware, no previous study has tried to identify adequate housekeeping genes for use in human heart tissue. Morgan $e t$ al. [15] examined the expression of genes related to the renin-angiotensin system in human atrial tissue using GADPH as an endogenous control. Other authors [16] have used RNA extracted from human endomyocardial biopsies and isolated cardiomyocytes for real-time RTPCR using GADPH, HPRT and the oncogene ABL as housekeeping genes. These endogenous controls showed very low variation in individual gene expression levels 
across cardiac pathologies, suggesting their suitable use as reference genes for quantitative PCR studies in cardiac tissue. In these previous studies, the specific testing of several candidate reference genes to determine the most suitable reference for use in cardiac tissue was not reported $[15,16]$. In contrast, Radonic et al. [17] determined transcription levels of several housekeeping genes in different human tissues, including heart, and identified TBP as the gene with the lowest range of RNA transcription across tissues. This finding is in agreement with the present results.

We found PPIA, RPLP plus GADPH to be a reliable set of genes for normalising data (Figure 3 ) according to the geNorm programme. As reported by Vandesompele et al. [6], geNorm proposes a pairwise variation of 0.15 as the cut-off under which the inclusion of an additional control gene is not required. Using our set of candidate genes, this would mean that adding a fifth gene to the four most stable genes identified would really provide the best results. Notwithstanding, this cut-off of 0.15 should not be considered in a strict sense, but rather as a reference to determine the optimal number of housekeeping genes. Sometimes the observed trend can be equally informative, and using the three best reference genes is, in most cases, a valid strategy for much more accurate and reliable normalisation compared to the use of a single housekeeping gene.

Using NormFinder software [8] as another tool to validate the expression stability of the ten candidate reference genes; GADPH, PPIA and RPLP also achieved the best stability values. While geNorm detected the two genes whose expression ratios showed least variation from those of the other genes tested, NormFinder was able to identify the single gene with the most stable expression. Hence, the most stable candidate gene was found to be GADPH, and the least stable TBP. Using this programme, we obtained the same results as with GeNorm except for the rank position ascribed to GADPH as the most stable single gene. However, the least stable genes and most stable ones identified by GeNorm and Normfinder were generally wellmatched.

As a limitation to our study, we should mention that although we established the purity of our RNA samples, due to the amount of RNA available, we could not run electrophoretic tests to check RNA integrity.

Finally, the set of reference genes determined here as the best endogenous controls to be used in qRT-PCR studies in heart tissue has applications in developing cardiovascular diagnostic tests and therapeutic strategies that will substantially improve human health [18].

\section{Conclusion}

It is commonly accepted that gene expression studies should be normalised using more than one reference gene. Based on our results, we propose the use of the mean result rendered by PPIA, RPLP and GADPH as housekeeping genes to normalise gene expression values obtained by qRT-PCR in heart tissue.

\section{Methods \\ Sample collection}

Tissue samples were obtained from the left ventricle of 35 organ donors (19 male, 16 female; mean age 59 years, range $40-80$ years). The organ donors had died in a multidisciplinary ICU of a tertiary university hospital. In $85 \%$ of the organ donors, brain death was attributed to a non-traumatic cause and no donor had previous heart pathology. Consent for organ donation for transplant and clinical investigation was obtained from the relatives of each donor.

The hearts had been rejected for transplant because of donor age or weight, or recipient blood group incompatibility. The cardiac tissues were freshly harvested, stored immediately in O.C.T ${ }^{\mathrm{TM}}$ Compound (Tissue Tek, Sakura, Netherlands) and then frozen at $-80^{\circ} \mathrm{C}$.

\section{RNA extraction and CDNA synthesis}

Isolation of total RNA was performed using the Nucleo Spin RNA II Isolation Kit (Macherey-Nagel Gmb H \& Co. $\mathrm{KG}$ ) according to the manufacturer's instructions.

The quality of the RNA was assessed through absorbance measurements made using a NanoDrop ND-1000 UV-Vis Spectrophotometer (NanoDropTechnologies, Wilmington, DE, USA). All the samples used in the PCR procedures showed a $260 / 280 \mathrm{~nm}$ absorbance ratio between 1.8 and 2.2. A ratio of $\approx 2$ is generally accepted as pure for RNA.

Each RNA sample was reverse transcribed using the OligopdT and Random primers provided in the first-strand complementary DNA (cDNA) synthesis kit for RT-PCR (AMV; Roche Diagnostic, Switzerland) following the manufacturer's instructions. The tubes were incubated at $25^{\circ} \mathrm{C}$ for 10 minutes to allow annealing. Reverse transcription was conducted at $42^{\circ} \mathrm{C}$ for 60 minutes, followed by 5 minutes incubation at $99^{\circ} \mathrm{C}$ to denature the enzyme. The cDNA samples were then cooled at $4{ }^{\circ} \mathrm{C}$ and stored at $-20^{\circ} \mathrm{C}$ until use.

To confirm the total absence of residual DNA, we performed a conventional PCR with $\beta$-actin [GenBank:E00829] primers designed using Beacon Designer software (Premier Biosoft International, California) spanning intron-exon boundaries ( $\beta$-actin up 5'-GACTACCT- 
CATGAAGATCCTC-3'; $\quad \beta$-actin down 5'CGGATGTCCACGTCACACTTC-3'). The PCR protocol consisted of an initial denaturation step at $94^{\circ} \mathrm{C}$ for 5 minutes, followed by 35 amplification cycles. Each cycle involved a denaturation step of 30 seconds at $94^{\circ} \mathrm{C}$, followed by 30 seconds of primer annealing at $55^{\circ} \mathrm{C}$ and 30 seconds of primer extension at $72^{\circ} \mathrm{C}$ followed by a final extension of 10 minutes. Amplicon size was confirmed by electrophoresis using an ethidium bromide-stained 2\% agarose gel in 1XTBE buffer.

\section{Gene expression}

We analysed the expression of the genes GADPH, PPIA, ACTB, YWHAZ, RRN18S, B2M, UBC, TBP, RPLP and $H P R T$ included in the Human Endogenous Control Gene Panel supplied by TATAA Biocenter (Goteborg, Sweden) (see Table 2). For this panel, the genes are carefully selected to be of different functional classes, thus significantly reducing the likelihood that they might be co-regulated. The genes selected here were also evaluated by Vandesompele et al. [6].

Quantitative PCR was performed using an i-Cycler iQ Real-Time PCR Detection System (Bio-Rad, Hercules, CA, USA). SYBR Green PCR Supermix (2X) (Bio-Rad), which detects amplification using Sybr Green as a doublestranded DNA-specific fluorescent dye, was used as a mastermix. Assays were performed in duplicate and a blank included in every assay. The reaction mixture for amplification consisted of $2 \mu \mathrm{l}$ of cDNA in a final reaction volume of $25 \mu \mathrm{l}$, as indicated in the instructions for the Human Endogenous Control Gene Panel. The RT-PCR protocol included an initial step of $95^{\circ} \mathrm{C}$ ( 3 minutes), followed by 40 cycles of 30 seconds at $95^{\circ} \mathrm{C}$ for DNA denaturation, 20 seconds for primer annealing at $60^{\circ} \mathrm{C}$ and 20 seconds at $72^{\circ} \mathrm{C}$ for primer extension. Fluorescence data were acquired at $72^{\circ} \mathrm{C}$. Melting-curve analysis to confirm product specificity was performed immediately after amplification, following 1 minute denaturation at $95^{\circ} \mathrm{C}$, 1 minute annealing at $65^{\circ} \mathrm{C}$ and 60 cycles of $0.5^{\circ} \mathrm{C}$ increments ( 10 seconds each) beginning at $65^{\circ} \mathrm{C}$ while monitoring fluorescence. Product identity was confirmed by electrophoresis using an ethidium bromide-stained 2\% agarose gel in 1XTBE buffer.

PCR efficiencies were calculated from a relative standard curve derived from a pool of heart tissue specimens from the same organ donors and serially diluted (a ten-fold dilution series with at least six measuring points).

\section{Reference gene expression stability}

To determine the most stable housekeeping genes of the set of tested genes in our heart cDNA sample panel, we used firstable, the geNorm software package for Microsoft Excel $^{\oplus}$ developed by Vandesompele et al. [6]. The programme geNorm uses an algorithm to calculate $M$, a gene expression stability measure, defined as the mean pairwise variation for a given gene compared to the remaining tested control genes. Thus, the higher the $M$ value of a gene, the greater the variation in its expression levels. The programme establishes a rank order of gene stability via stepwise exclusion. To determine how many reference genes should be used, normalisation factors $\left(\mathrm{NF}_{\mathrm{n}}\right)$ based on the geometric mean of the expression levels of the $n$ best reference genes were calculated by stepwise inclusion of an extra, less stable reference gene as described elsewhere [4]. The programme shows the pairwise variation $\mathrm{Vn} / \mathrm{V}_{\mathrm{n}+1}$ between two sequential normalisation factors, $\mathrm{NFn}$ and $\mathrm{NF}_{\mathrm{n}+1}$. A large variation means that the added gene has a significant effect and should probably be included for calculation of the normalisation factor.

To compare our results with those generated by a similar programme, we used Normfinder [9], another algorithmbased tool for identifying optimal reference genes among a set of candidates. The programme ranks the genes depending on their expression stability value in given samples derived from an experimental design.

\section{Authors' contributions}

The first author of the manuscript SP performed the experimental procedures. LJR, AR and EG provided support for the qRT-PCR and discussion. AA and FA harvested the heart tissue. DE was the main person responsible for obtaining tissue samples from organ donors. The project

Table 2: Abbreviations and functions of the genes mentioned in the text

\begin{tabular}{ccc}
\hline GADPH & Glyceraldehyde-3-phosphate dehydrogenase & Glycolytic enzyme \\
PPIA & Peptidylprolyl isomerase A (cyclophilin A) & Immunity protein \\
ACTB & Actin beta & Cytoskeletal structural protein \\
YWHAZ & Tyrosine 3/tryptophan 5-monooxygenase activation protein, zeta polypeptide & Protein signal transduction \\
RRN18S & I8S rRNA & Ribosomal RNA \\
B2M & Beta-2-microglobulin & Beta-chain of major histocompatibility complex class I molecules \\
UBC & Ubiquitin C & Protein degradation \\
TBP & TATAA box binding protein & General RNA polymerase II transcription factor \\
RPLP & 60S Acidic ribosomal protein P0 & Member of the ribosome proteins \\
HPRT & Hypoxanthine phosphoribosyltransferase & Purine synthesis
\end{tabular}


was designed by JO. All the authors have read and approved the final manuscript.

\section{Acknowledgements}

This research was supported by grant IB05-I35 from the Ficyt. We also thank to the Spanish Ministry of Science and Education (project NO AGL2005-04479).

\section{References}

I. Ginzinger DG: Gene quantification using real-time quantitative PCR: an emerging technology hits the mainstream. Exp Hematol 2002, 30(6):503-5I2.

2. Schmittgen TD, Zakrajsek BA: Effect of experimental treatment on housekeeping gene expression: validation by real-time, quantitative RT-PCR. J Biochem Biophys Methods 2000, 46(I2):69-8I.

3. Gorzelniak K, Janke J, Engeli S, Sharma AM: Validation of endogenous controls for gene expression studies in human adipocytes and preadipocytes. Horm Metab Res 200I, 33(I0):625-627.

4. von Smolinski D, Leverkoehne I, von Samson-Himmelstjerna G, Gruber AD: Impact of formalin-fixation and paraffin-embedding on the ratio between mRNA copy numbers of differently expressed genes. Histochem Cell Biol 2005, I24(2): 177-I88.

5. Suzuki T, Higgins PJ, Crawford DR: Control selection for RNA quantitation. Biotechniques 2000, 29:332-337.

6. Vandesompele J, De Preter K, Pattyn F, Poppe B, Van Roy N, De Paepe A, Speleman F: Accurate normalization of Real-Time quantitative RT-PCR by geometric averaging of multiple internal control genes. Genome Biol 2002, 3(7):34.

7. Tricarico C, Pinzani P, Bianchi S, Paglierani M, Distante V, Pazzagli M, Bustin SA, Orlando C: Quantitative real-time reverse transcription polymerase chain reaction: normalization to rRNA or single housekeeping genes is inappropriate for human tissue biopsies. Anal Biochem 2002, 309:293-300.

8. Andersen CL, Jensen JL, Orntoft TF: Normalization of real-time quantitative reverse transcription-PCR data: a model-based variance estimation approach to identify genes suited for normalization, applied to bladder and colon cancer data sets. Cancer Res 2004, 64(I 5):5245-5250.

9. NormFinder Software [http://www.mdl.dk/publication snormfinder.htm]

10. SAS/STAT TM, 1999. User's Guide. Release 8.2. SAS Institute Inc, 703 Cary NC. 704.

1I. Thellin O, Zorzi W, Lakaye B, De BB, Coumans B, Hennen G, Grisar $T$, Igout $A$, Heinen $E$ : Housekeeping genes as internal standards: use and limits. J Biotechnol 1999, 75:29I-295.

12. Glare EM, Divjak M, Bailey MJ, Walters EH: Beta-Actin and GADPH housekeeping gene expression in asthmatic airways is variable and not suitable for normalising mRNA levels. Thorax 2002, 57:765-770.

13. Yperman J, De Visscher G, Holvoet P, Flameng W: Beta-actin cannot be used as a control for gene expression in ovine interstitial cells derived from heart valves. J Heart Valve Dis 2004, 13(5):848-853.

14. Bustin SA, Nolan T: Pitfalls of quantitative real-time reverse transcription Polymerase Chain Reaction. J Biomol Tech 2004, 15:155-166.

15. Morgan K, Wharton J, Webb JC, Keogh BE, Smith PL, Taylor KM, Oakley CM, Polak JM, Cleland JG: Co-expression of renin-angiotensin system component genes in human atrial tissue. J Hypertens Suppl 1994, I 2(4):SII-19.

16. Moniotte S, Vaerman J-L, Kockx MM, Larrouy D, Langin D, Noirhomme P, Balligand J-L: Real-Time RT-PCR for the detection of beta-adrenoceptor messenger RNAs in small human endomyocardial biopsies. J Mol Cell Cardiol 200I, 33:2I II-2I33.

17. Radonic A, Thulke S, Mackay IM, Landt O, Siegert W, Nitsche A: Guideline to reference gene selection for quantitative realtime PCR. Biochem Biophys Res Commun 2004, 3 I 3:856-862.

18. Seo D, Ginsburg GS, Goldschmidt-Clermont PJ: Gene expression analysis of cardiovascular diseases: novel insights into biology and clinical applications. J Am Coll Cardiol 2006, 48(2):227-235.
Publish with Biomed Central and every scientist can read your work free of charge

"BioMed Central will be the most significant development for disseminating the results of biomedical research in our lifetime. "

Sir Paul Nurse, Cancer Research UK

Your research papers will be:

- available free of charge to the entire biomedical community

- peer reviewed and published immediately upon acceptance

- cited in PubMed and archived on PubMed Central

- yours - you keep the copyright 